\title{
Multilinguales
}

\section{Jalons pour une mise en visibilité de la sexualité dans Triptyque de Claude Simon: discursivité, voies de fait et dispositifs}

Milestones for a visualization of sexuality in Triptyque by Claude Simon: discursivity, ways of fact and devices

\section{Sinan Anzoumana}

\section{OpenEdition \\ Journals}

Édition électronique

URL : http://journals.openedition.org/multilinguales/3893

DOI : $10.4000 /$ multilinguales.3893

ISSN : 2335-1853

\section{Éditeur}

Université Abderrahmane Mira - Bejaia

\section{Référence électronique}

Sinan Anzoumana, « Jalons pour une mise en visibilité de la sexualité dans Triptyque de Claude

Simon : discursivité, voies de fait et dispositifs », Multilinguales [En ligne], 11 | 2019, mis en ligne le 17 juillet 2019, consulté le 03 juin 2020. URL : http://journals.openedition.org/multilinguales/3893 ; DOI : https://doi.org/10.4000/multilinguales.3893

Ce document a été généré automatiquement le 3 juin 2020

\section{(c) (†) $\ominus$}

Multilinguales est mise à disposition selon les termes de la Licence Creative Commons Attribution Pas d'Utilisation Commerciale - Pas de Modification 4.0 International 


\title{
Jalons pour une mise en visibilité de la sexualité dans Triptyque de Claude Simon : discursivité, voies de fait et dispositifs
}

\author{
Milestones for a visualization of sexuality in Triptyque by Claude Simon: \\ discursivity, ways of fact and devices
}

Sinan Anzoumana

1 La sexualité et sa pratique féconde ont été de tous les temps au cœur de l'évolution de l'humanité. Selon Foucault, elle s'apparente à un :

[...] grand réseau de surface où la stimulation des corps, l'intensification des plaisirs, l'incitation au discours, la formation des connaissances, le renforcement des contrôles et des résistances, s'enchaînent les uns avec les autres, selon quelques grandes stratégies de savoir et de pouvoir (1976:139).

2 La question ne relèverait plus du domaine du sacré comme le sexe l'était à ses origines jusqu'à une certaine période (Antiquité et Moyen Âge); il est de plus en plus profané et déversé dans l'espace de la connaissance, du savoir. Il investit à juste titre, sans condition, l'espace public, les domaines de l'activité créatrice en particulier la littérature. Dans un article, Pierre N'Da examinant son intrusion dans l'espace littéraire stipule que :

Comme pour affirmer le règne et l'omniprésence du sexe, comme pour proclamer, sans fausse pudeur, le triomphe du sexe, les écrivains ne veulent plus se taire ni se cacher derrière les mots. Ils parlent ouvertement, effrontément de ce qui, habituellement et par décence, est tu, réservé, tenu secret, caché, interdit, c'est-àdire défendu de dire, ou ce qui ne doit pas être dit entre gens en public, au risque de déranger l'ordre des choses et la bienséance. (2011)

3 À la suite de N'Da, Olivier Bessard-Banquy affirme que " [l]e sexe a naturellement colonisé les pages [de la littérature]»(14). Ainsi, au regard d'une certaine relation entre la construction d'un fantasme sexuel et sa mise en spectacle sur une page blanche, le sexe 
ne s'affirme pas uniquement en tant que paradigme thématique ; il développe de même et dans une certaine mesure une discursivité sexuelle selon Anne Juranville qui soutient que : "C'est [un] discours commun sur le sexe, qui ne correspond évidemment pas nécessairement à des pratiques sociologiquement repérables [...]» (2007: 19). Dès lors, ce qui nous intéressera dans cette présente analyse, c'est la perspective profane, ostentatoire et ostensible du sexe développée à partir du XX $\mathrm{XX}^{\mathrm{e}}$ siècle. À cette période, se développent et se divulguent toutes sortes d'images érotico-pornographiques dont le flot échappe à toute tentative de censure. Pour Christophe Genin,

Ces images pornographiques sont bien ostensibles au sens où elles montrent qu'elles montrent. Elles sont emphatiques au sens où elles insistent sur leur volonté

de faire spectacle en sollicitant, par exemple, le regard du spectateur (2013:10).

$\mathrm{Au}$ regard des textes de Claude Simon, il apparaît que Triptyque entretient un tel dessein. Il sera donc, à point nommé, un support favorable à prospection en ce sens que la discursivité du sexe se manifeste non seulement dans et par la scripturalité, mais également par l'usage de procédés qui immergent totalement le lecteur dans l'érotisme et la pornographie. S'inscrivant de fait dans le sillage des théories foucaldiennes de la sexualité, ce présent argumentaire tentera d'appréhender l'espace de la sexualité et son entour dans l'imaginaire littéraire simonien. Autrement dit, comment se construit le rapport entre forme narrative et formes de sexualité dans Triptyque? Quels procédés littéraires sont employés dans l'intention d'entraîner le lecteur-spectateur spécifiquement dans un univers imaginaire sexuel? Enfin, quelle esthétique découlerait d'une telle mise en écriture du sexe et de la sexualité ?

Pour ce faire, après avoir questionné en premier lieu la rhétorique simonienne en usage dans Triptyque, l'étude s'attachera en second lieu à montrer les dispositions formelles textuelles qui emportent et immergent le lecteur dans une sorte de voyeurisme intégral. En dernier lieu, l'analyse montrera comment l'écriture du bas-corps en action ici induit une véritable esthétique de l'oxymore.

\section{Tout dire : procédés narratifs et réalisme des descriptions}

6 Annie Clément-Perrier stipule qu'" Écrire [le sexe], c'est l'écrire sous tous ses aspects, dans ses lumières et dans ses ombres " (1999: 39). Autrement dit, le sexe doit s'appréhender dans sa dimension à la fois sacrée et profane, dans sa beauté comme dans sa laideur. Or le pari de certains écrivains néo-romanesques, postmodernes surtout africains, c'est d'exceller dans l'écriture de la profanation et de la vulgarisation du sexe et de son entour en démystifiant les métadiscours afférents. À ce propos, Pierre N'Da stipule que :

[Les écrivain(e)s] parlent du sexe, sans retenue aucune ; ils[elles] décrivent le sexe, crûment; ils[elles] dépeignent, avec force détails et sans maquillage, des scènes érotiques ou pornographiques, des séances d'orgies sexuelles; ils présentent complaisamment des sexualités déviantes, interdites, transgressives, désordonnées, débridées ou libérées. (2011)

7 À la suite de N'Da, Alain Ricard soutient qu'écrire le sexe, c'est " ...écrire ce qu'il ne faut pas écrire. [...] les écrivains écrivent comme il ne faut pas écrire » (1995 : 234). Claude Simon, écrivain iconoclaste, ayant adopté cette posture scripturale offre à travers Triptyque (Simon, 1973) un texte teinté d'ambiguïté sexuelle : en même temps que le récit flirte 
avec la pornographie ${ }^{1}$, il s'inscrit également dans l'érotisme. ${ }^{2}$ Apparemment, cette forme de monstration s'enracine résolument dans un champ lexical approprié.

\section{Un champ lexical sexuel du cru et de l'ostentatoire}

8 Anne-Charlotte Millepied déclare que «[1]e vocabulaire constitue un [des traits] du régime discursif [érotico-pornographique] : il est spécifique, car "plutôt proscrit dans les situations sociales ordinaires" »(2014: 4). En effet, les multiples séquences descriptives dans Triptyque illustrent fort bien son propos :

[...] D'un violent coup de reins la fille se dégage de l'étreinte de l'homme couché sur elle et dont le membre glissant hors de la vulve inonde de sperme les cuisses, le ventre et le pubis aux poils noirs, se tendant violemment, presque à l'horizontale, à chaque éjaculation, le gland très rouge et luisant se gonflant chaque fois comme s'il allait éclater. Le sperme laiteux coule sur la peau blanche et le long de l'aine de la fille qui reste immobile, raidie, les jambes maintenant étendues, la tête renversée et tournée sur le côté, comme morte, le corps pesant allongé sur elle, comme mort lui aussi (66).

9 L'acte mimétique de l'acte coïtal se transcrit ici grâce à un vocabulaire cru, ordinaire et surtout très précis. Pour Pierre Caminade cité par Stéphanie Orace, il s'agit plutôt de » mots sensuellement choisis» (2009: 140). Dès lors, les indicateurs textuels verbaux "glissant, inondent ...», adjectivaux « laiteux, violent ...», adverbiaux "violemment ... », les syntagmes nominaux « membre, vulve ... » qui foisonnent dans le tissu textuel permettent de présumer une phénoménalité érotico-pornographique pour aller dans le sens d'oliver Aïm (Aïm, 2013: 15-20). Ainsi, tout en se voulant plus explicite et expressif, le vocabulaire de Triptyque ne se sublime pas dans l'euphémique; au contraire, le mot employé en contexte appelle immédiatement l'image à l'esprit. Cette force de Triptyque, Madeleine Chapsal l'évoque dans sa revue de presse :

Certains morceaux sont d'une extrême crudité un valet de ferme culbute une servante sur un tas de foin, et les détails anatomiques et physiologiques de l'opération sont donnés avec tout autant de précision visuelle que lorsqu'il s'agit du corps d'une charrette, du bâti de la grange ou du rais de lumière venu de la lucarne où se pressent deux têtes de gamins rieurs. (L'Express, 1973)

10 On l'aura compris, chez Simon, la description n'est pas étagée, tout apparaît logé à la même enseigne; il en va ainsi pour la précision dans la description d'objet banal que pour le sexe humain. Tout est dit impassiblement, sur le même ton et dans le même ordre. Il n'y a pas de tabous pour Claude Simon, tout se décrit de la même manière. L'auteur n'émet pas de jugement et évite de verser dans une sorte d'hiérarchisation entre ce qui peut se dire. Bref, autant une pomme est une pomme, autant un sexe est un sexe. Les mots circulent donc chez Simon au sein d'une description dynamique et surtout productive au sens ricardolien, description qui s'adosse sur la puissance de ces mots à effet visuel. En outre, il y a un glissement de vocabulaire d'une description à l'autre de sorte que la sexualité envahit et contamine même les autres éléments du texte :

La fente dans l'affiche déchirée ne permet de voir que la partie centrale des deux corps coupés à la taille par le bord de droite, c'est-à-dire la hanche de la femme, sa fesse, sa cuisse repliée, [...] (20). (Nous soulignons)

Puis, plus loin, on peut lire :

[...], le périnée où court déjà une légère mousse de poils blonds qui se fait plus épaisse de chaque côté de la fente de la vulve lilas qui s'entrouvre sur une ligne d'un rose vif (37). (Nous soulignons) 
11 Loin donc des expressions raffinées, les réalités du sexe et de la sexualité sont dites, exposées sans détour à l'instar d'une pathologie dont on présente les symptômes sans vocabulaire dramatique ou spécialisé. Pour reprendre Juranville, il s'agit chez l'écrivain "[...] d'exposer, sans états d'âme et sans fioritures, ce qui relève du plus intime de l'univers sexuel [...]»(2007: 24). Ce vocabulaire du cru, de la "réalité », disséminé dans la description simonienne est presque l'acte fait dans l'écriture. Pour preuve :

[...] Se maintenant sur l'autre jambe et appuyée du dos contre le mur de briques, elle a relevé sa cuisse repliée contre son ventre et introduit en elle d'une main le membre raidi de l'homme dont la peau parait d'autant plus blanche, comme phosphorescente elle aussi, [...]. Pour mieux pénétrer la fille l'homme a légèrement fléchi les genoux (Simon, $1973: 21$ ).

12 La description érotico-pornographique s'exprimant ainsi dans Triptyque l'est à travers le pouvoir des mots à vouloir décrire exactement des scènes sexuelles ou à propension sexuelle. Ainsi, les mots renvoient le plus directement possible à la chose et se substituent presque à elle. Conséquence, les descriptions produisent des effets de réel sur le lecteur dans la mesure où les descriptions des scènes sont données pour vraies avec les mots les plus crus, les expressions les moins cachées. Au bout du compte, le dynamisme de Triptyque réside dans son pouvoir à faire évoluer des scènes subjectives vers des scènes objectives. Or, comme le stipule Camille Dreyfus-Le Foyer

[...] pour être pornographique l'image devrait se méfier d'un simulacre et de l'illusion. Elle devrait se présenter comme une image qui ne simule pas, mais déjoue les pièges de l'apparence, de l'image elle-même. Bref, comme une image qui dépasse la représentation pour se faire pure présentation, exhibition. L'image pornographique serait une image déjouant les pièges de l'imaginaire $(2010: 10)$.

Du reste, la question de la langue étant fondamentale chez l'auteur, elle se pose même au-delà du simple vocabulaire. Selon Alastair Duncan, "Les signifiés de son matériau dépassent également le mot : ils consistent non pas simplement en "vocabulaire", mais aussi en "figures", "tropes", et finalement en "les éléments d'une fiction" » (2006: 32).

\section{Métaphorisation vs sexualisation : expansion de facteurs communs ${ }^{3}$}

14 Si la dimension ostensible et ostentatoire du récit est avérée, il n'en demeure pas moins une écriture hautement métaphorisée. Le tissu analogique y est comme un labyrinthe et au bout, une textualité sexuelle finit par devenir une sexualité textuelle. Selon Catherine Haman-Dhersin,

Le texte est saturé d'images ambiguës; sous l'effet des premiers émois amoureux des deux adolescents, les paysages les plus neutres en apparence se sexualisent, les faisceaux d'évocations juxtaposées composent une toile à forte coloration libidinale (2012:161).

15 Cette ambiguïté scripturale, Ralph Sarkonac le souligne en affirmant que «... le sexe relève tantôt de l'explicite, tantôt de l'implicite. D'ailleurs, on pourrait voir, dans ce rapport, l'image même, la métaphorisation textuelle de la relation sexuelle" (Sarkonac, 2009: 49). En s'appuyant sur le texte, on note le développement d'une sémantique lexicale ressortie aux liens implicites qu'entretient le déroulement de l'écriture avec l'acte sexuel, liens que Sarkonac qualifie de "non-dits». Le jeu des métaphores apparait ici essentiel au point de susciter et favoriser, selon Duncan, les passages entre les scènes de fiction : « [la] prolifération d'analogies élargit énormément la gamme des transitions possibles entre les scènes de fiction » (2006: 39). Bien qu'elles 
soient foisonnantes les analogies, nous prendrons toutefois, à titre d'illustration, un seul rapprochement, celui entre la femme et la vache :

L'épaisse toison noire où le membre luisant continue son va-et-vient contraste avec la blancheur lisse des fesses et des cuisses. [...] Cinq ou six vaches que pousse devant lui un gamin à la chevelure filasse sommairement coupée à coups de ciseaux sur la nuque traversent le pont d'un pas lent, leurs hanches osseuses oscillant avec raideur. Leur pelage est roux avec de larges taches blanches (Simon, 1973 : 16-17).

À l'analyse, la femme et la vache sont effectivement reliées corporellement par les syntagmes nominaux « les cuisses », « les fesses » qui deviennent du coup des facteurs communs entre la femme et la vache. Ensuite, un lien cette fois chromatique entre les deux prend forme à travers les facteurs communs «blancheur/blanches». Dans cette combinatoire, l'homme tisse également des liens avec le règne animal, précisément le léporidé. En l'occurrence, les syntagmes adjectivaux «luisant» et "raidi» qui qualifient «membre » semblent exprimer le degré de virilité du sexe masculin; d'où la métaphore de l'homme-lapin. D'ailleurs, ce sexe sera par la suite comparé à un serpent ${ }^{4}$. Selon l'expression de Ralph Sarkonak, il s'agit d'« une très phallique vipère » $(2009: 44)$ :

[...] les yeux effrayés fixés sur quelque chose de noir et luisant dressé dans l'herbe de la berge là où ils étaient l'instant d'avant, la tête plate à angle droit tourné dans leur direction, [...] (Simon, $1973: 153$ ).

Enfin, en s'intéressant au couple adultérin, l'idée immédiate d'une réification de celuici qui l'identifie à des objets inertes comme le matériel agricole (p. 43), la motocyclette (p. 49) est, en réalité, balayée par l'effet d'une sensualité qui contamine tous les objets présents dans l'espace où se déroule l'acte amoureux :

[...] accédant tout à coup à une dimension solennelle, définitive, comme si les personnages avaient été tout à coup plaqués contre quelque muraille invisible et transparente, pris au piège dans l'air brutalement solidifié, passant d'un instant à l'autre à l'état d'objets inertes, choses parmi les choses qui les entourent sur la surface de l'écran. [...] (194-195)

18 Somme toute, ces multiples métaphores jouent en réalité de la médialité entre le règne humain, animal, végétal et minéral. Hormis ce jeu métaphorique combinatoire, le texte brouille une métaphorisation sexuelle par contiguïté fondée sur une construction cataphorique :

Quoique ni l'un ni l'autre n'ait parlé la truite file sur la droite d'un rapide coup de queue et se glisse dans l'ouverture du broc couché sur le flanc. Avant qu'elle disparaisse les garçons ont pu voir briller son ventre clair. Lorsque le bassin de l'homme recule on entrevoit pendant une fraction de seconde son membre luisant et cylindrique sortant à demi de l'épaisse toison noire entre les cuisses repliées, presque bleues, comme du lait, $[. . .]^{5}(15)$.

La proximité sémantique entre les syntagmes " rapide » et " une fraction de seconde »; «l'ouverture du broc » et «toison noire »; «briller » et » luisant »; « se glisse dans » et » sortant à demi»; «ventre clair» et «les cuisses [...] lait» préfigure l'acte de copulation à venir même si dans la séquence sexuelle qui suit immédiatement, la description est brouillée par des mouvements contraires. En tout état de cause, de façon subtile et bien articulée, l'acte textuel sublime l'acte sexuel et vice versa. Comme le laisse entendre Haman-Dhersin, "[les] connotations sexuelles [des] extraits sont d'autant plus manifestes qu'[ils] sont immédiatement précédés [de] scènes de coït» (2012: 168). Triptyque présente ainsi un double enjeu érotico-pornographique dans ce sens que les nombreuses métaphores filées formant de vastes systèmes analogiques érotisent 
excessivement le texte. À rebours, le système des cataphores pornographise le récit selon les termes de Olivier Aïm. En outre, le texte est sous-tendu par une violence qui collabore parallèlement avec les actes sexuels du récit.

\section{Le « crash », le « trash » et le « gore » en œuvre}

Anne Juranville fait savoir que :

...Le caractère banalisé, surexposé du sexe, inséparable de l'escalade de la représentation de la violence - le «crash», le «trash», le "gore» ... -, est un phénomène sociologique inédit qui tient d'abord au système médiatique toutpuissant dans lequel nous vivons (2007: 19).

Pour assoir alors une vraie matrice évènementielle, dramatique à Triptyque, il a fallu que l'auteur y ajoute aux séquences sexuelles explicites du crash, du trash, du gore. Ainsi, ces séquences de violence, de crime et autres servent de liants, suturant chacune des séquences sexuelles entre elles. Dans cette perspective, nous avons par exemple l'évocation de la mise à mort du lapin qui est immédiatement suivie de la séquence du sexe tendu de l'amant de la grange. Alastair Duncan cité par Catherine Haman-Dhersin écrit à ce sujet: $»$ Par sa proximité textuelle, cette lame brillante du couteau semble menacer autant le membre luisant de l'homme que la vie du lapin" (2012: 163). Triptyque révèle en fait deux sources de violence; l'une externe provenant de la mise à mort du lapin, externe à l'acte sexuel qui sert de liant à la trame narrative érotico-pornographique pauvre et l'autre, interne à l'acte, exprimée par l'homme sur la femme. Cette dernière forme de violence est exprimée par des syntagmes adjectivaux « violent(e)s, rugueux/ rugueuse(s)»; nominaux "violence »; adverbiaux "violemment». Cette violence ${ }^{6}$ masculine est mise en abyme dans une affiche programmatique (p. 95) dont une clé de lecture sera fournie par le narrateur lui-même à la page 175 à propos de cet homme : " ...comme s'il n'était capable que d'actions incontrôlées et violentes... ». Foucault parle alors de «psychiatrisation du plaisir pervers » (1976: 139) induisant du coup la figure de l'adulte pervers dans ce texte. C'est à juste titre que N'Da soutient encore que :

[...] le sexe et la violence s'imbriquent souvent et explosent dans des scènes érotiques ou pornographiques qui aboutissent à des actes ignobles de meurtre. [...] les atrocités inhumaines et déshonorantes [...], montrent à quel point, dans certaines circonstances de la vie, l'homme, livré à ses instincts et assuré de toutes représailles, devient plus animal qu'un animal féroce, plus pervers et plus sadique qu'un monstre, débile mental (2011).

En fin de compte, le crime et la violence qui circulent dans ce texte servent de prétextes pour brouiller une liaison narrative sexuelle très pauvre, devenant ainsi la matrice fondamentale sur laquelle s'adossent et se développent les séquences sexuelles. Au fond, les scènes érotico-pornographiques dans Triptyque ne viennent pas ponctuer, en marquant ses temps les plus frétillants, un récit qui les rassemblerait, les légitimerait, c'est au contraire ce récit de violence, de crime, secondaire qui essaie de donner une illusion de cohérence à l'ensemble en recousant chacune de ces scènes sexuelles entre elles. Du reste, ces scènes érotico-pornographiques tirent leur puissance expressive du dispositif qui les soutient. 


\section{Tout montrer et tout voir : dispositifs cinématographiques}

La prégnance de techniques cinématographiques dans Triptyque apparaît en adéquation avec la rhétorique érotico-pornographique en usage. Nous convenons dès lors avec Aïm pour soutenir que Triptyque devient " [...] l'objet paradigmatique d'une histoire des supports, des écrans et des visualités " (2013: 16). Concrètement, la matérialité du cinéma prend forme ici à travers les affiches, les mouvements de la caméra ainsi que les différents plans de prise de vue. Selon Claude Mauriac,

Plus que d'un puzzle, il s'agit de pellicule en vrac dont le montage complexe révèle, sous le désordre apparent, un ordre subtil. Livre fait de films, films qui font le livre. Films soit projetés dans deux cinémas [...]. Travellings arrière ou avant, panoramiques. La caméra se substitue au regard des spectateurs, qu'ils soient dans le film, qu'ils regardent le film ou qu'ils lisent le livre. Le metteur en scène et l'auteur ne font qu'un. Les éléments cinématographiques sont à mesure transformés en matière littéraire. (Le Figaro, 1973)

Des éléments métaphoriques préfigurant le cinéma circulent dans le texte et viennent corroborer cette indication paratextuelle. D'abord l'eau, élément reflétant la lumière qui est consubstantielle au cinéma. Ensuite, le train en tant métaphore de mouvement. Pour Irène Albers,

[le cinéma] y est présent sous tous ses aspects, en tant que prise de vue dans un studio, positions et mouvements d'une caméra, affiches de cinéma, narrations mélodramatiques, projections dans des salles de cinéma, bouts de pellicule isolés, etc. (Albers, $2006: 309$ ).

À côté de ces métaphores cinématographiques, la représentation du plus intime trouve dans les techniques cinématographiques un support idéal.

\section{(Très) gros plan : en quête du voir}

Selon Bérénice Bonhomme,

Claude Simon privilégie les gros plans dans les descriptions de scènes d'amour [...] L'ambigüité du gros plan érotique est encore plus évidente dans Triptyque où nous est décrit un film pornographique. Le corps apparaît comme morcelé, obscène et monstrueux (2010: 184-185).

Or, le principe même du porno, c'est le gros plan qui permet d'aller au plus près du détail. Selon Lucien Dällenbach, ...avec ses gros plans, le texte simonien, en réalité, en est à mille lieues : montrer le sexe - tout le sexe- en disant qu'on le montre, et qu'on le montre au même titre qu'autre chose, c'est ne pas en faire une "histoire " et en tout cas, ne pas donner dans le « cochon» (1988: 71).

La dimension sensuelle du texte se résoudrait finalement ici dans l'ostensible et dans un souci du détail. C'est d'ailleurs la raison pour laquelle Triptyque use aussi du très gros plan dans l'intention du détail anatomique du génital et des scènes coïtales. Au fond, le très gros plan infère le morcellement qui accentue davantage la place du génital :

...le membre de l'homme couché en travers de l'aine commence à bouger, animé de faibles tressaillements qui le font d'abord rouler sur le haut de la cuisse, puis se gonfle et durcit, s'allongeant par une série d'infimes saccades. Entourée par la 
couronne plissée du prépuce, on aperçoit la pointe rose du gland, percé de son œil aveugle (Simon, $1973: 188$ ).

Victor Bachy, dans Universalis, affirme que :

Les moments de suspense d'un film éveillent un sentiment d'attente angoissée. Le suspense est fondé sur la probabilité de la solution plus ou moins prochaine d'une situation ambiguë. Il va jouer sur la durée et peut être tragique; il joue aussi sur la peur $[\ldots]$

i l'on s'en tient au fait qu'en tant que technique narrative, le suspens(e) précède la surprise, et que son efficacité réside dans un dosage adéquat de la durée et de la pertinence des éléments qui créent la sensation d'attente angoissante ; si le suspense se veut une émotion caractérisée par une incertitude liée au développement anticipé d'un événement inachevé alors la fragmentation et le morcellement du texte au niveau structurel, adossés sur une construction parataxique induisent alors un effet de suspens(e). À partir de cette portion de texte,

$\mathrm{Au}$ bout de l'un d'eux, [...] pend un lapin au pelage gris perle tenu par les oreilles, tantôt parfaitement immobile, tantôt agité de soubresauts et de coups de reins impuissants. Sortant de l'autre main aux doigts noueux et jaunes on peut voir par instants briller la lame de couteau. La fille couchée dans le foin accompagne de coup de reins le va-et-vient rythmé des fesses de l'homme dont on voit chaque fois briller le membre luisant qui disparaît ensuite jusqu'aux couilles entre les poils touffus, noirs et brillants [...] (25),

la sensation de peur finit par se convertir en plaisir sexuel. En outre, le suspens(e) se manifeste également dans les transitions de la trame narrative : une scène sexuelle se voit brusquement figée en un objet iconique ${ }^{8}$. De même, une scène inanimée se voit soudainement saisie de vitalité et projetée sur un écran de cinéma99.

En définitive, nous convenons toujours avec N'Da qui formule que :

La littéralité d'un texte, on le sait, ne se situe pas dans le contenu du discours, ni dans la valeur morale et la convenance des termes choisis, ni même dans la qualité de la syntaxe; elle est plutôt dans l'écart, dans la démarcation par rapport aux 
normes habituelles, dans la transgression et la subversion de la langue, dans la manière singulière, anomale et anormale de s'exprimer, dans la façon poétique, artistique ou esthétique de dire ou d'écrire sa pensée. Quoi de plus littéraire que le roman du sexe ou l'écriture de la sexualité pour parler du sexe, pour exprimer la sexualité et la sensualité, la beauté et le charme du corps ? (2011)

Triptyque s'inscrit dans cet écart, dans cette distance vis-à-vis de la normale en épousant tout à fait la pensée foucaldienne :

Le sexe, selon la nouvelle pastorale, ne doit plus être nommé sans prudence; mais ses aspects, ses corrélations, ses effets doivent être suivis jusque dans leurs rameaux les plus fins [...] tout doit être dit (1976:28).

Le sexe et son entour deviennent ainsi scène par l'entremise des technicités cinématographiques. Cette union entre cinéma, érotisme et pornographie inscrit sans doute Triptyque dans l'oxymorique. De plus, l'écriture du sexe et ses corrélations apparaissent ici comme une stratégie de discursivation et surtout de délégitimation des métadiscours (Lyotard, 1979: 8). Il s'agit chez l'auteur de démystifier, de désacraliser le sexe pour aller dans le sens de Juranville :

«Comme si le même sillon que creusaient tous ces écrits consistait à faire une littérature sur le sexe qui démystifie le sexe : en le banalisant, en le désacralisant (Millet) ; en le naturalisant (Reyes) ; en le simplifiant (Ernaud) ». (2007: 25)

Triptyque parodie de la sorte l'hypocrisie du langage institutionnel, délégitimant tout discours officiel sur la sexualité. Le sexe devient un savoir qui doit être débarrassé des tabous, des interdits, des verrous sociaux et des traditions pour dévoiler ses secrets, pour démasquer et exprimer haut et fort la réalité du sexe vécue au quotidien. Et ce faisant, on pourra inscrire ce texte au nombre des sextuels selon López García : « La fusion des corps textuel et sexuel, que nous nommons "sextuel", se caractérise par la fragmentation, par l'hybridité, le travestissement, l'emphase, l'exagération... ${ }^{10} »(2010: 17)$.

\section{BIBLIOGRAPHIE}

AÏM, Olivier, « De la pornophonie à la pornographie », Proteus, 5, 2013, p. 15-20. Disponible sur : (www.revue-proteus.com/articles/Proteus05-2.pdf), (consulté le 24/01/19).

ALBERS Irène, NITSCH Wolfram, Transports : les métaphores de Claude Simon, Bruxelles, Peter Lang, 2006.

BACHY, Victor, « Entrée de Suspense », Cinéma, Encyclopcedia Universalis. Disponible sur : (http:// www.universalis.fr/encyclopedie/suspense-cinema/), (consulté le 24/01/19).

BESSARD-BANQUY, Olivier, Le livre érotique, Bordeaux, Presses Universitaires Bordeaux, 2010.

BONHOMmE, Bérénice, Claude Simon : une écriture en cinéma, Berne, Peter Lang, 2010.

, Claude Simon, la passion cinéma, Villeneuve-d'Ascq, Presses Universitaires du Septentrion, 2011.

CHAPSAL, Madeleine, « Triptyque », Les Éditions de Minuit. Disponible sur : (http://

www.leseditionsdeminuit.fr/livre-Triptyque-1859-1-1-0-1.html), (consulté le 24/01/19). 
CLÉMENT-PERRIER, Annie, « Le Jeu des couleurs dans Le Jardin des Plantes ». dans Littératures 40, printemps 1999. Claude Simon : Le Jardin des Plantes, Toulouse, Presses Universitaires du Mirail, 1999, p. 31-46.

DÄLLENBACH, Lucien, Claude Simon, Paris, Seuil, 1988.

DREYFUS-LE FOYER, Camille, L’X (pornographie), Paris, M-Editer, 2010.

DUNCAN, Alastair B., "Claude Simon, Michel Deguy et Triptyque », dans ALBERS Irène, NITSCH Wolfram, Transports : les métaphores de Claude Simon, Bruxelles, Peter Lang, 2006, p. 31-41. foUCAUlT, Michel, Histoire de la sexualité I. La volonté de savoir, Paris, Gallimard, 1976.

GENIN, Christophe, « Entre l'industrie de l'Internet et la solitude des graffiti », in Proteus, 5, 2013, p. 6-14. Disponible sur : (www.revue-proteus.com/articles/Proteus05-2.pdf), (consulté le 24/01/19).

HAMAN-DHERSIN, Catherine, Paysages de Claude Simon, Villeneuve d'Asq, Septentrion, 2012. JURANVILLE, Anne, « » L'érotisme en question. » Regard sur quelques aspects de la littérature féminine contemporaine », in Connexions, 87, 2007, p. 19-42. Disponible sur : (http:// www.cairn.info/revue-connexions-2007-1-page-19.htm), (consulté le 24/01/19).

LOPEZ, Garcia, Isabelle, « Du genre pornographique aux signes épidémiques du corps dans Loco Afán de Pedro Lemebel », in Lectures du Genre, 7, 2010. Disponible sur (http:// www.lecturesdugenre.fr/Lectures_du_genre_7/Lopez-Garcia.html), (consulté le 24/01/19). , La question du genre dans les chroniques de Pedro Lemebel, Milagros Ezquerro (dir.), Thèse de doctorat, Université de Paris-Sorbonne Paris IV, 12 novembre 2007.

LYOTARD, Jean-François, La condition postmoderne, Paris, Minuit, 1979.

MAURIAC, Claude, « Triptyque », Les Éditions de Minuit. Disponible sur (http:// www.leseditionsdeminuit.fr/livre-Triptyque-1859-1-1-0-1.html), (consulté le 24/01/19).

MILLEPIED, Anne-Charlotte, « Marie-Anne Paveau, Le discours pornographique », in Lectures, 30 septembre 2014. Disponible sur (https://journals.openedition.org/lectures/15638), (consulté le 24/01/19).

N'DA, Pierre, « Le sexe romanesque ou la problématique de l'écriture de la sexualité chez quelques écrivains africains de la nouvelle génération », in Éthiopiques, 86, 2011. Disponible sur (http:// ethiopiques.refer.sn/spip.php?article1759), (consulté le 24/01/19).

ORACE, Stéphanie, « Désir du rythme, rythme du désir : autour d'un éventail », in ILIAS YOCARIS (dir.), Sofistikê, n 1, 2009, p. 133-163. Disponible sur www.sofistike.fr (consulté le 24/01/19) RICARD, Alain, Littérature d'Afrique noire : des langues aux livres, Paris, CNRS- Karthala, 1995. RoLIN, Dominique, « Triptyque », Les Éditions de Minuit. Disponible sur (http:// www.leseditionsdeminuit.fr/livre-Triptyque-1859-1-1-0-1.html), (consulté le 24/01/19). SARKONAK, Ralph, "Comment noyer le poisson, ou le "non-dit" dans Triptyque », in ILIAS YOCARIS (dir.), Sofistikê, 01, 2009, p. 37-60. www.sofistike.fr (consulté le 24/01/19) SIMON Claude, Triptyque, Paris, Minuit, 1973. 


\section{NOTES}

1. Les séquences descriptives sexuelles du texte donnent dans l'ostensible et l'ostentatoire.

2. En effet, au moment où l'on pense que l'imagination est anéantie parce que devinant l'enchainement logique de l'acte, la suite du texte, dans une forme de collage, fait surgir une séquence descriptive autre qui entretient l'évocation, le mystère, la sensualité, caractéristiques de l'érotisme.

3. Le facteur commun est une loi mathématique. En Arithmétique, il s'agit d'un nombre qui peut diviser exactement deux ou plusieurs nombres entiers différents (ex : 3 est un facteur commun de 6 et de 9). Il existe des correspondances entre 3 et $6(6=3 \times 2)$, entre 3 et $9(9=3 \times 3)$ ou des échos : 3 est en écho dans 6 et 9. On pourra aussi dire que 3 est non-dit dans 6 ou 9, ou dissimulé dans ces deux nombres.

4. Il s'agit sans doute de la taille du sexe de l'homme, sexe que les enfants-voyeurs commenteront plus tard.

5. Le syntagme nominal «coup de queue » chez le poisson sera substitué par «coup de reins » chez l'homme (Triptyque, p. 25).

6. Cette violence rapproche l'homme de l'animal: divisé en deux parties, l'une humaine - qui semble avoir complètement disparu - et l'autre animale qui contrôle ses volontés et ses gestes (intelligence et rapidité en même temps qu'indifférence).

7. Le principe de loupe est proche de celui du (très) gros plan.

8. Il s'agit entre autres de la carte postale, la gravure, l'affiche ou les illustrations de magazine.

9. Ces séquences peuvent être lues aux pages 94-95, 194-195.

10. Pour plus de précision, voir Lopez Garcia Isabelle, La question du genre dans les chroniques de Pedro Lemebel, Milagros Ezquerro (dir.), Thèse de doctorat, Université de Paris-Sorbonne Paris IV, 12 novembre 2007.

\section{RÉSUMÉS}

Articulé autour de la mise en visibilité de la sexualité, cet argumentaire aborde l'examen du désir et la représentation du sexe au cinéma dans Triptyque de Claude Simon sous les couples voir/savoir et voiler/dévoiler, c'est-à-dire un désir d'exactitude et de connaissance par un effort de clarté totale ou au contraire par le refus de toute part d'ombre au compte de la pure transparence et qui engage une discursivation de Triptyque de l'ob-scène à la scène. Cela immerge le lecteur dans une sorte de voyeurisme au cœur d'une fictionnalité travaillée par le dolorisme. Reposant sur l'illusion mimétique, cette stratégie discursive mise en place est censée donner pour vraie une représentation du sexe et de la sexualité. Montrant finalement son propre fonctionnement, Triptyque marque en ce qu'il remet au jour « l'incrédulité à l'égard des métarécits ».

Articulated around the visibility of sexuality, this argument deals with the examination of desire and the representation of sex in cinema in Claude Simon's Triptych under the couples to see / know and to veil / unveil, that is to say a desire of exactness and knowledge by an effort of total clarity or, on the contrary, by the refusal of any shadow to the account of pure transparency and which engages a discourse of Triptych from the ob-stage to the scene. This immerses the reader in a sort of voyeurism at the heart of a fictionality worked by dolorism. Based on the mimetic illusion, this discursive strategy put in place is supposed to give a true representation of sex and 
sexuality. Finally showing its own functioning, Triptych marks, in that it brings to light "unbelief with regard to meta-narratives".

INDEX

Keywords : writing, speech, devices, sexuality, representation

Mots-clés : écriture, discours, dispositifs, sexualité, représentation

\section{AUTEUR}

\section{SINAN ANZOUMANA}

Université Félix Houphouët-Boigny, Côte d'Ivoire 